\title{
Stress lass nach! - Was ist eigentlich Stress?
}

\author{
Volker Schmiedel
}

Fühlen Sie sich gestresst? Selbstverständlich sind Sie gestresst. Sie können es sich heute gar nicht mehr leisten, nicht gestresst zu sein. Selbst wenn Sie nicht gestresst sein sollten, dann würden Sie das niemals zugeben, weil Sie das in unserer Leistungsgesellschaft äußerst suspekt macht - entweder lügen Sie dann oder Sie sind ein fauler Hund.

\section{Definition}

Der Begriff „Stress“ kommt aus dem Englischen und bedeutet Druck oder Anspannung. Ursprünglich stammt er aus dem Lateinischen, hier wird „stringere“ mit anspannen übersetzt. Eigentlich stammt die Bezeichnung „Stress“ aus der Werkstoffkunde, bei der man unter Stress eine Materialermüdung durch Zug oder Druck auf ein Material versteht. Hans Selye war es, der 1936 den Stress in die Biologie einführte. Er definierte ihn als „unspezifische Reaktion des Körpers auf jegliche Anforderung“. Als Stressor bezeichnet man einen Reiz, der eine solche unspezifische Reaktion auszulösen vermag.

Stress ist also nicht der Stau, in dem wir stehen, wenn wir zu einem Notfall müssen, der nörgelnde Kunde oder die Steuererklärung - das alles sind Stressoren. Es sind Reize, die Stress auslösen können. Wenn wir aufgrund eines oder mehrerer Stressoren anfangen zu schwitzen, wenn der Blutdruck steigt oder dafür prädestinierte Menschen Harndrang bekommen - das ist dann der eigentliche Stress.

\section{Physiologie und Evolutionsbiologie}

Wir wollen uns nicht mit biologischen Stressreaktionen wie Kälte - Aufrichten der Körperhaare (Gänsehaut) oder bakte- rielle Infektion - Fieber (Symptom einer Immunreaktion) beschäftigen, sondern mit psychischem Stress.

Ein Stressor - wie das Klingeln des Telefons, die verletzende Kritik des Teilhabers oder der Gedanke des Anfangsassistenten an einen Notfall - führt zur Ausschüttung von Stresshormonen wie Adrenalin, Noradrenalin oder Cortisol aus der Nebenniere. Diese wiederum bewirken u.a. einen kurzfristigen Blutdruckanstieg, einen erhöhten Blutzuckerspiegel und die Freisetzung von Fettsäuren.

Stress wird landläufig immer mit etwas Negativem in Verbindung gebracht. Weit gefehlt! Stress ist lebensnotwendig für uns. Unsere Vorfahren hatten die Fähigkeit, auf Anforderungen oder Stressoren mit körperlichen und seelischen Antworten zu reagieren. Mit der Aktivierung des Kreislaufs, dem erhöhten Zuckerund Fettsäurespiegel im Blut waren unsere Vorfahren in der Lage, körperliche Höchstleistungen zu vollbringen: Sie erlegten das Mammut oder rannten vor dem Säbelzahntiger davon. Diejenigen unserer Vorfahren, die fähig waren, richtig guten Stress zu entwickeln, überlebten. Die anderen starben aus.

Wir alle sind Nachfahren gestresster, aber überlebender Höhlenmenschen, und uns unterscheidet wirklich erstaunlich wenig von ihnen. Unsere biologische Ausstattung ist nahezu dieselbe. Eines aber unterscheidet uns tatsächlich: die Regenerationsfähigkeit. Nach erfolgreicher Jagd auf das Mammut gab es erstmal einen Festschmaus. Man schlug sich den Bauch voll, und dann wurde geruht. Wozu erneut jagen, wenn noch was vom Mammut übrig war? In dieser Ruhephase sanken die Spiegel der Stresshormone im Blut. Das vegetative Nervensystem schal-

\section{.konkret}

Das Gefährliche ist nicht der Stress selbst, sondern, dass wir ihn nicht in körperliche Leistung umsetzen und dass wir nicht zur Ruhe kommen, den Stress ausklingen lassen und in der Regenerationsphase die Energiereserven wieder auffüllen. Nicht der Stress führt zum Burnout, Diabetes oder Herzinfarkt, sondern der inadäquate Umgang damit!

tete von Aktivität auf Ruhe, der Körper konnte regenerieren.

Und genau diese Regenerationsphasen fehlen uns heute. Nach einem stressigen Arbeitsalltag setzen wir uns nicht etwa hin, freuen uns des Daseins und bauen Stress ab. Nein, wir wollen sofort das nächste Mammut erlegen. Nach Möglichkeit sollte es auch noch größer sein als das vorige. Aus dem rhythmischen Auf und $\mathrm{Ab}$ von Aktivität und Passivität, Stress und Regeneration wird eine bedrohliche Stress-Spirale, die sich immer schneller dreht.

Ein weiterer wichtiger Unterschied besteht zwischen dem Stress der Urmenschen und dem des heutigen Homo sapiens. Wenn wir Stress haben, dann sollen wir kämpfen oder fliehen (auf neudeutsch: fight or flight). Der Stress dient hauptsächlich der Fähigkeit, körperliche Leistungen zu entwickeln. Unsere körperlichen Reaktionen beim heutigen Stress beschränken sich aber darauf, auf das Gaspedal zu drücken (flight) oder die Autohupe zu betätigen (fight), wenn uns der Vordermann auf der Autobahn geärgert hat. Physiologisch sinnvoller wäre es, zum nächsten Parkplatz zu fahren und dort den Stress mit einem $1 / 2$-stündi- 
gen Waldlauf abzubauen. Homo sapiens heißt übrigens weiser Mensch, worüber sich durchaus diskutieren ließe.

\section{Gesunder Stress?}

Es geht gar nicht darum, jeden Stress zu vermeiden - nicht jeder Stress ist schlecht. Wenn wir das Siegtor unserer Fußballmannschaft bejubeln, so bezeichnen wir dies als Eustress, also als guten Stress. Ohne ein wenig Stress könnten wir eine anspruchsvolle Aufgabe, z.B. einen Vortrag oder eine Examensprüfung, weniger konzentriert und erfolgreich bewältigen. Eine Überreaktion auf Stress kann natürlich ebenfalls kontraproduktiv sein.

Stress ist gefährlich, wenn er dauerhaft anhält oder so schnell wiederkehrt, dass wir keine ausreichende Möglichkeit zur Regeneration haben.

Dann können Krankheiten wie Bluthochdruck oder Fettstoffwechselstörungen mit den möglichen Folgen eines Herzinfarkts oder eines Schlaganfalls resultieren. Wenn wir ihn jedoch „abarbeiten“, dann bleiben auch seine schädlichen Folgen aus. Unnötigen Stress sollten wir uns daher - wenn irgend möglich - ersparen.

\section{Diagnostik - objektive Messmethoden}

Leider gibt es kein direktes Verfahren, um Stress sicher und quantifizierbar zu messen. Die Stressfolgen (z.B. Auswirkungen auf Blutdruck, Blutzucker und Blutfette) kann man natürlich messen, wobei Stress hierbei nur eine mögliche Ursache darstellt, wenn auch häufig die wichtigste.

Ein relativ neues Verfahren ist die HRVMessung. Dabei wird die Herzratenvariabilität (HRV) gemessen. Aus den unterschiedlichen Abständen zwischen den einzelnen Herzaktionen können mithilfe einer besonderen Software wichtige Informationen gewonnen werden. Die Aktivität von Sympathikus und Parasympathikus kann dabei quantitativ bestimmt werden. Bei Stress herrscht eine Dominanz des Sympathikus, des aktivieren- den Teiles des vegetativen Nervensystems. Kann der Parasympathikus als beruhigender Teil des vegetativen Nervensystems hier nicht ausgleichen, kommt es zu einer Dysbalance. Dieser überwiegt in der Regel in der Nacht. Lässt sich zu wenig Parasympathikusaktivität nachweisen, kommt es zu einem wenig erholsamen Nachtschlaf, einem häufigen Phänomen bei chronischem Stress. Entspannungsphasen am Tag, z.B. durch Meditation oder Mittagsruhe, können ebenso nachgewiesen werden wie die Länge und Häufigkeit von Tiefschlafphasen. Diese Resultate werden anhand von Zahlen und Grafiken überzeugend dargestellt. Die Notwendigkeit ausgleichender Maßnahmen wie Sport oder Entspannungsübungen kann somit unterstrichen werden. Und das Beste: Nach einer Umsetzung der Empfehlungen kann der Erfolg für jeden sichtbar dokumentiert werden.

Des Weiteren messe ich bei gestressten Personen gerne:

- Magnesium („Anti-Stress-Mineral“) im Vollblut

- Q10 („Energie-Aktivator“) im Serum

- TSH (Schilddrüsenhormon als Zündkerze des Energiestoffwechsels) im Serum

- Hormone im Speichel

Eine umfassende Diagnostik beinhaltet für mich eine Hormonanalyse aus dem Speichel. Das Cortisol kann bei chronischem Stress im Tagesprofil erhöht sein. Bei lang andauerndem Stress habe ich auch erniedrigte Werte gefunden, die dann aber bereits mit einer spürbaren Erschöpfung verbunden waren. Unterschiedliche Cortisol-Werte haben natürlich auch andere Therapiestrategien zur Folge. Bei Erschöpfung messe ich auch DHEA (Dehydroepiandrosteron, eine Vorstufe von Östrogen, Progesteron und Testosteron), bei Schlafstörungen zusätzlich Melatonin.

\section{Sinnvolle Therapie}

Das Natürlichste bei der Stressbewältigung ist die körperliche Bewegung. Schließlich werden die Stresshormone genau dafür gebildet. Körperliche Arbeit verstärkt selbst aber auch noch einmal deren Freisetzung - je intensiver, desto mehr. Am günstigsten zum Abbau der Stressfolgen sind - je nach Leistungsfähigkeit - moderate Ausdauersportarten wie Spaziergänge, Walking, Jogging, Radfahren und Schwimmen. Diese Sportarten sollten mindestens 3-mal in der Woche und mindestens $\mathbf{3 0}$ Minuten am Stück durchgeführt werden. Dabei sollte ein Belastungsniveau gewählt werden, das es uns noch erlaubt, das eine oder andere Wort zu sprechen („Laufen ohne zu Schnaufen“). Genauer ist natürlich eine Leistungsdiagnostik mit Belastungs-EKG, Spiroergometrie oder Laktatmessung, um die genauen Herzfrequenzen für bestimmte Belastungsbereiche zu berechnen.

Der andere Königsweg zum Stressabbau sind Entspannungsverfahren. Dies können Achtsamkeitsübungen wie etwa der Bodyscan sein, die man sogar während der Arbeit in wenigen Sekunden oder Minuten anwenden kann. Darunter fallen Entspannungsübungen wie Yoga, autogenes Training, Muskelentspannungstraining nach Jacobson oder Tai Chi. Diese Methoden können wir mittlerweile mithilfe von Kursen (z.B. Volkshochschule, Animation in Ferienanlagen, Reha-Kliniken), Büchern, einer CD oder im Internet (z. B. www.youtube.de) erlernen.

\section{Motivation}

„Das Rationale am Menschen sind die Einsichten, die er hat. Das Irrationale an ihm ist, dass er nicht danach handelt“ (Friedrich Dürrenmatt, Schriftsteller, 1921-1990).

Das Problem ist dabei die Motivation. Gerade diejenigen, die Entspannungsübungen am nötigsten haben, entwickeln die größte Abneigung dagegen. Gerade „rationale Macher-Typen“ wehren sich am meisten. Sie werden auf ihren Stress erst aufmerksam, wenn der Arzt ihnen ein Antihypertensivum verschreibt oder wenn sie nach einem Herzinfarkt auf einer Intensivstation aufwachen. Das ist wirklich irrational, denn die Effekte von Entspannungsverfahren sind mittlerweile wissenschaftlich gut erforscht. Die Wirkungen auf Blutdruck, Fettstoffwechsel und deren Folgen stehen denen 
"richtiger“ Medikamente nicht nach ohne aber mit deren Nebenwirkungen behaftet zu sein.

Die eigene Haltung zu bestimmten Situationen ist unglaublich wichtig für das Ausmaß des Stresses. Wenn ich eine Situation akzeptiere oder eine Tätigkeit gerne ausübe, dann ist dies weit weniger stressend als wenn ich dagegen eine Aversion entwickele. Tun Sie also das, was Sie tun, mit Vergnügen. Sollte dies in überwiegendem Maße - nicht der Fall sein, sollten Sie vielleicht etwas anderes tun. Denn Rudolf Steiner (1861-1925) sagte einmal:
„Es gibt kaum etwas Schlimmeres, als wenn man seelisch mit seinem Herzen dem fern steht, was der Kopf treiben muss.“

\section{Fazit}

Mit einer differenzierten und subtilen Diagnostik können adäquate therapeutische Maßnahmen in die Wege geleitet werden. Dies ist - zusammen mit notwendigen Veränderungen des Lebensstils - eine ganzheitliche und natürliche Behandlung von Stress.
Online zu finden unter

http://dx.doi.org/10.1055/s-0033-1345518

\section{Literatur}

1 Schmiedel V. Burnout - wenn Arbeit, Alltag \& Familie erschöpfen. Stuttgart: TRIAS; 2010

\section{Dr. med. Volker Schmiedel, MA}

Chefarzt der Inneren Abteilung der Habichtswald-Klinik

Wigandstr. 1

34131 Kassel Bad Wilhelmshöhe 